\title{
Aflatoxina em fígados de frangos de corte, com esteatose, abatidos industrialmente no Estado do Rio de Janeiro
}

\section{Aflatoxin in broiler chickens livers free to use in the State of Rio de Janeiro}

\author{
Rosana Rangel Duarte', Eulógio Carlos Queiroz de Carvalho², Carlos Alberto R. Rosa ${ }^{3}$
}

\section{Resumo}

Visando a detecção de aflatoxina $\mathrm{B} 1$ em fígados de frangos de corte liberados para consumo, foi realizada coleta de amostras direcionada pela presença de características macroscópicas indicativas de esteatose (palidez e/ou coloração amarelada) hepática na linha de retirada das vísceras em uma indústria sob inspeção estadual no município do Rio de Janeiro, Estado do Rio de Janeiro. Após a liberação para consumo e antes da lavagem e resfriamento com gelo, dentro de lotes de 4.000 aves, as amostras foram separadas em seis grupos de acordo com um intervalo de freqüência da característica escolhida. A histopatologia evidenciou esteatose em quatro dos seis grupos, sendo detectada aflatoxina B1 em três dos seis grupos.

Palavras chave: aflatoxina B1; fígado de aves; esteatose

\section{Introdução}

A presença de aflatoxina (AFT) em grãos destinados ao consumo não é fato raro, tendo sido relatado por Sylos e Rodrigues-Amaya (1994) que a encontraram em amendoim cru e torrado. Nos produtos destinados à alimentação animal, foi detectada aflatoxina B1 (AFB1) em 451 das 1.131 amostras analisadas, inclusive com níveis superiores a $30 \mathrm{ppb}$, chegando a um máximo de 10.807 ppb (Baldissera et al., 1994). Essa micotoxina é, portanto, um contaminante de vários ingredientes de rações, especialmente cereais, estando relacionada com várias condições patológicas no homem e nos animais.

Em aves, a AFB1 produz fígados aumentados, amarelados e friáveis, hemorragia petequial, marcada vacuolização de células hepáticas e proliferação de dutos biliares, como observado por Hamilton e Garlich (1971) e Edds et al. (1973), podendo o diagnóstico presuntivo da aflatoxicose em aves que consumiram rações contami- nadas ser baseado no aumento do fígado, somado ao seu aspecto pálido e friável (Huff e Doerr, 1981; Trucksess et al., 1983; Balachandran e Ramakrishnan, 1987; Micco et al., 1988; Mahalinghan et al., 1989; Johri et al., 1990), mesmo em animais tratados que não exibam sinais clínicos, mortalidade ou outras lesões macroscópicas.

A AFB1 e seus biotransformados podem ser encontrados no fígado e em outros tecidos comestiveis de aves (Mabee e Chipley, 1973), durante um tempo que varia em função da quantidade ingerida (Sawhney et al., 1973; Trucksess et al., 1983; Obioha et al., 1986; Micco et al., 1988; Fernández et al., 1994). Micco et al. (1988), concluem que aves tratadas com baixas doses de AFB1 por longo tempo não são rejeitadas no momento da inspeção de carnes, mas que resíduos deAFB1 permanecem presentes nos tecidos comestíveis, podendo ser encontrado também o aflatoxicol (AFL) em pequena proporção nas aves.

A presença de AFB1 em tecidos de animais abatidos para consumo foi objeto da pesquisa de Sabino et al. (1994) que analisaram 83 amostras de fígados e rins de suínos e aves colhidas aleatoriamente, nas quais encontraram apenas traços de aflatoxina M1 (AFM1) em uma única amostra de rins das aves e AFB1 em uma única amostra de fígado de suíno.

Uma situação que pode agravar o já exposto é sugerida no trabalho de Wyatt et al. (1987) que mostraram ser possível a seleção de aves cada vez mais resistentes à AFB1, obtendo uma queda acentuada da mortalidade com altas doses de AFB1 em gerações sucessivas (cinco gerações) de frangos de engorda comerciais.

Este trabalho tem como objetivo detectar a presença daAFB1 em fígados de aves abatidas para consumo, elegendo as amostras a serem analisadas com base numa característica macroscópica de fácil inspeção e sugestiva de aflatoxicose: 0 aspecto pálido e amarelado do fígado das aves.

\footnotetext{
${ }^{1}$ Este trabalho é um desdobramento de tese de Mestrado em Higiene Veterinária e Processamento Tecnológico de P.O.A (Aves) da primeira autora na UFF.

2 Setor de Patologia Veterinária, Universidade Federal Fluminense, UFF, Rua Vital Brasil Filho 64, 24230-340 Niterói, RJ, Brasil

${ }^{3}$ Departamento de Micologia e Micotoxicologia, DMIV, Universidade Federal Rural do Rio de Janeiro (UFRRJ), km 47 da Antiga Rodovia RioSão Paulo, 23851-970 Seropédica, RJ, Brasil
} 


\section{Material e Métodos}

As amostras foram colhidas, dentre os fígados liberados para consumo, em um estabelecimento industrial, sob inspeção estadual, localizado no município do Rio de Janeiro, Estado do Rio de Janeiro, durante o processamento normal das aves. Cada lote foi composto por aves originárias de granjas diferentes. Verificou-se a freqüência do aspecto eleito em aproximadamente 350 fígados, colhendo-se aproximadamente $500 \mathrm{~g}$ de fígados pálidos. As amostras foram identificadas e divididas nos seguintes intervalos: de menos de $1 \%$ até $3 \%( \pm 2 \%)$, acima de $3 \%$ até $6 \%( \pm 5 \%)$ e acima de $6 \%( \pm 8 \%)$, constituindo os grupos especificados na Tabela.

Tabela - Divisão das amostras colhidas por grupos, de acordo com dia da coleta, ordem de coleta e freqüência (\%) de palidez (P)

\begin{tabular}{cccccc}
\hline $04 / 10$ & $\mathrm{P}(\%)$ & $11 / 10$ & $\mathrm{P}(\%)$ & $17 / 10$ & $\mathrm{P}(\%)$ \\
\hline $\mathrm{A}$ & \pm 8 & $\mathrm{~A}$ & \pm 8 & $\mathrm{~A}$ & \pm 8 \\
$\mathrm{~B}$ & \pm 5 & $\mathrm{~B}$ & \pm 2 & $\mathrm{~B}$ & \pm 2 \\
\hline
\end{tabular}

O material para a histopatologia foi separado e fixado em formol-salina neutra a $10 \%$. O restante foi mantido congelado para posterior determinação de AFTs.

As amostras para o estudo morfohistoquímico foram processadas por inclusão em parafina e coradas pela hematoxilina-eosina ( $\mathrm{HE}$ ), além de cortadas em criostato e coradas pelo Sudam-III. Este método foi utilizado para a evidenciação de gordura, quando sua presença era sugerida pela $\mathrm{HE}$.

Os padrões de AFB1, AFM1 e AFL utilizados foram adquiridos da Sigma Co. (USA), padronizados e calibrados segundo a metodologia descrita no Manual de Métodos Oficiais de Análises da AOAC seção 971.22 (AOAC, 1990).

A metodologia empregada para a obtenção do extrato e sua purificação foi baseada naquela descrita por Trucksess e Stoloff (1984) e modificada por Sabino et al. (1995) para fígado in natura. O desenvolvimento dos extratos foi realizado tanto por cromatografia em camada delgada de sílica gel (CCD) como por cromatografia líquida de alta eficiência (CLAE) usando-se coluna de fase normal (sílica) e coluna de fase reversa de octadecilsilano (C18).

Os equipamentos e colunas utilizados para a detecção foram: bomba marca Waters, mod. 510; detectores UVVIS, marca Merck-Hitachi, mod. L-4250 e de fluorescência, marca Waters, mod. 420 A-C; integrador/registrador, marca Merck-Hitachi, mod. D-2500; injetor, marca Water, mod. U6K; detector de arranjo de diodo, marca Merck-Hitachi, mod.L-3000 acoplado a microcomputador AT-286/s e com software DAD-6000 Merck-Hitachi e sis- tema de compressão radial, marca Waters, mod. RC-100.

As fases estacionárias (placas de sílica, pré-colunas e colunas) compreenderam A: cromatofolhas de sílica gel 60G-Merck (No. 5533); B: sílica gel 80G Merck (No. 7734); C: coluna radial Pak Si $10 \mathrm{~m}$ e coluna radial Pak $\mathrm{C} 18$ (Waters), Hibar C18 $5 \mathrm{~m}$ Merck e respectivas pré-colunas (Waters e Merck).

A confirmação da identidade das AFT e seus biotransformados foi realizada por derivação com TFA/ nHexano (AFB1) e por análise do espectro UV de cada uma delas em sistemas cromatográficos diferentes, comparados aos dos padrões por analisados em detector de arranjo de diodo.

Para o estabelecimento dos coeficientes de recuperação e o limite de detecção de AFB1, AFM1 e AFL, soluções padrões destas micotoxinas foram adicionadas às amostras de fígado e, após a evaporação do solvente dos padrões, as amostras fortificadas foram submetidas à metodologia analítica e quantificadas por CCD e CLAE. Foram feitas três repetições para cada uma das concentrações de toxinas adicionadas às amostras .

Análises histopatológicas de amostras de fígado dos grupos 04/10-A, 04/10-B, 11/10-A e 17/10-A revelaram, na $H E$, vacúolos citoplasmáticos de diferentes dimensões que, submetidos ao método de Sudam III, revelaram a natureza lipídica de seus conteúdos. Raramente, de permeio, hepatócitos com citoplasma tumefeito e/ou com formações vacuolares de limites mal definidos e não sudanófilas eram notados, sugerindo desequilíbrio hídrico intracelular (degeneração hidrópica) .

AFB1 foi detectada, por CLAE, em três amostras de fígado de aves (grupos 04/10 A; 04/10 B e 17/10 B) nas concentrações de 1,2, 1,8 e 3,2 ng/g"1 (ppb), respectivamente, conforme demonstrado nos cromatogramas $4,5 \mathrm{e}$ 6. AFM1 e AFL não foram detectados. No restante dos grupos não foram detectadas AFTs.

A identidade das AFTs (B1 e M1) foi confirmada por derivatização com ácido trifluoroacético nas amostras e nos padrões. A identidade do AFL foi confirmada por coinjeção e cromatografia em três sistemas solventes diferentes. Os resultados obtidos são equivalentes à média aritimética de três determinações.

\section{Discussão}

É notável, no levantamento bibliográfico realizado, a menção da palidez do fígado como uma característica marcante na inspeção macroscópica da agressão causada pela AFT (Hamilton e Garlich, 1971; Edds et al. 1973; Nair e Simpson, 1973; Huff e Doerr, 1981; Trucksess et al., 1983; Balachandram e Ramakrishnan, 1987; Micco et al., 1988; Mahalinghan et al., 1989; Johri et al., 1990).

Com a presença daAFT determinada tanto em produtos para consumo humano (Sylos e Rodrigues-Amaya, 1994), quanto em produtos destinados à alimentação ani- 
mal (Baldissera et al., 1994), e sendo o período necessário para sua eliminação do organismo superior ao período de jejum exigido para o abate da ave (Mabee e Chipley, 1973; Sawhney et al. 1973; Vadhera e Baker, 1973; Trucksess et al., 1983, Obioha et al., 1986; Micco et al., 1988; Fernández et al., 1994), podemos supor ser possível a presença da micotoxina nos tecidos comestíveis de aves levados ao consumidor.

$\mathrm{O}$ fato de pequenas quantidades de AFTs, oriundas da contaminação natural de diferentes alimentos destinados tanto aos homens quanto aos animais, poderem causar os efeitos descritos, aumenta mais ainda a necessidade e a importância de estudos sobre os fatores que afetam a saúde animal e a saúde humana. Esta necessidade, com relação às aves, pode aumentar com diversos fatores como a alta concentração depositada nos fígados, associada à possibilidade de seleção natural das aves para uma maior resistência à AFT (Wyat et al., 1987), o que aumentaria ainda mais a quantidade de micotoxina presente no fígado no momento do abate, sem que disso se suspeitasse, já que, segundo Micco et al. (1988), podem não ser detectadas alterações macroscópicas que provoquem a rejeição, mesmo sendo encontrados AFB1 e AFL nos tecidos comestíveis. Com isto, podemos ver que esta micotoxina pode chegar ao homem tanto diretamente por ingestão de grãos contaminados (Sylos e Rodrigues-Amaya, 1994), quanto seus biotransformados em produtos de origem animal podem acabar tornandose parte da alimentação humana quando em produtos destinados à alimentação animal (Baldissera et al., 1994)

O direcionamento da coleta de amostras, baseada no aspecto pálido e/ou amarelado dos fígados, mostrou-se satisfatoriamente produtivo, sugerindo uma relação entre a AFT e o aspecto eleito, de fácil observação macroscópica durante o abate, já que foi possível a detecção de AFB1 em três dos seis lotes selecionados, enquanto que no único trabalho encontrado no período compreendido por este levantamento, realizado em amostras de fígados e rins de suínos e aves considerados próprios para consumo, a coleta aleatória realizada não permitiu a evidenciação da micotoxina nas amostras relativas às aves, exceto AFM1 em um rim (Sabino et al., 1994).

A determinação de parâmetros macroscópicos que, quando associados, permitiam a suspeita da presença da AFB1, auxiliando tanto a inspeção de produtos de origem animal quanto os produtores, que sofrem com perdas que não são primariamente atribuídas à AFB1, como um menor ganho de peso, maior morbidade e letalidade de doenças comuns à criação de aves, é uma necessidade, face aos riscos que a AFB1 pode representar para a saúde humana e animal, além da evitar a diminuição ou o encarecimento da produção de uma proteína nobre, como a carne de aves.

Face aos resultados obtidos, podemos concluir não ser o período de jejum anterior ao abate suficiente para a elimi- nação da aflatoxina que possa estar presente no fígado das aves, não havendo, nas quantidades encontradas de AFB1, alterações macroscópicas suficientes para provocarem a rejeição da ave ou do órgão durante a inspeção.

\section{Abstract \\ Aflatoxin in broiler chickens livers free to use in the State of Rio de Janeiro}

In order to detect aflatoxin B1 in broiler chickens livers free to use, with macroscopic characteristics of hepatic lipidic changes (pale and/or yellow) samples were colected of the viscera remove line at an industry under state inspection in Rio de Janeiro county, State of Rio de Janeiro. After liberation for consumption and before washing and chilling with ice, into batches of 4,000 birds, the sample were separated into six groups according to the frequence interval of the choosen characteristics. The histopathology showed lipidic changes in four, and aflatoxin B1 was detected in three.

Key words: aflatoxin B1; poultry livers; esteatosis

\section{Referências bibliográficas}

AOAC - Association of Official Analytical Chemists 1990. Official Methods of Analysis of the Association of Official Analytical Chemists. Willian Horwitz, Washington, $1094 \mathrm{pp}$.

Baldissera MA, Santurio JM, Mallmann CA, Almeida CAA, Kipper M, Souza Jr CE, Santos XC Camargo BS 1994. Aflatoxinas, Zearalenona e Ocratoxina A em Alimentos. Resultados de 1987 a 1993. Anais do I Congresso Latino-Americano de Micotoxicologia - VII Encontro Nacional de Micotoxinas. Rio de Janeiro 26 a 30 de setembro, p. 90-92.

Balachandran C, Ramakrishnan R 1987. An experimental study on the pathology of aflatoxicosis in broiler chicken. Indian Vet J 64: 911-914.

Edds GT, Nair KPC, Simpson CF 1973. Effect of aflatoxin B1 on resistance in poultry against cecal coccidiosis and Marek's disease. Am J Vet Res 3(6): 819-826.

Fernández A, Verde MT, Gascón M, Ramos JJ, Gómez J 1994. Aflatoxin and its metabolites in tissues from laying hens and broiler chickens fed with a contaminated diet. I Sci Food Agric 65: 407-414.

Hamilton PB, Garlich JD 1971. Aflatoxin as a possible cause of fatty liver syndrome in laying hens. Poultry Science 50: 800-804.

Huff WE, Doerr JA. Sinergism between aflatoxin and ochratoxin A in broiler chickens. Poultry Science 60: 550-555.

Johri TS, Sadagopan VR, Shrivastava HP, Majumdar S 1990. Effect of dietary aflatoxin an the performance of purebred broiler chicks. Indian J Animal Sciences 60(10): 1246-1248.

Mabee MS Chipley JR 1973. Tissue distribution and metabolism of aflatoxin B1-14C in broiler chickens. Applied Microbiol 25(5): 763-769.

Mahalingam RJ, Balachandran C, Punniamurthy N, Govindan $S$ 1989. Pathology of feeding aflatoxin detoxified feed in broiler chickens. Indian Vet 66: 1013-1015. 
Micco C, Miraglia M, Onori R, Brera C, Mantovani AL, loppolo A, Stasolla D 1988 . Long-term administration of low doses of mycotoxins to poultry. 1. Residues of aflatoxin B1 and its metabolites in broilers and laying hens. FoodAdtives and Contaminants 5(3): 303-308.

Obioha WI, Stahr HM, Kraft AA 1986. Distribution and effects of aflatoxin in chicken tissues after feeding radiolabeled (14C) aflatoxin B1. J Food Protection 49(10): 799-805.

Sabino M, Purchio A, Milanez TV 1994. Aflatoxinas B1, M1 e Aflatoxicol: extração e caracterização em tecidos de suínos e aves. Anais do I Congresso Latino-Americano de Micotoxicologia - VII Encontro Nacional de Micotoxinas. Rio de Janeiro, 26 a 30 de setembro, p.120-122.

Sabino M, Purchio A, Milanez TV 1995. Aflatoxin B1, M1 and aflatoxicol in tissues and urine os calves receiving aflatoxin. Food Additivies and Contaminants 12(3): 467-472.

Sawhney DS, Vadehra DV, Baker RC 1973. The metabolism of $14 \mathrm{c}$ aflatoxins in laying hens. Poultry Science 52:13021309.

Sylos CM, Rodrigues-Amaya DB 1994. Ocorrência simultanea de ácido ciclopiazônico e aflatoxinas $B$ e $\mathrm{G}$ em amendoim e milho. Anais do I Congresso Latino-Americano de Micotoxicologia - VII Encontro Nacional de Micotoxinas. Rio de Janeiro, 26 a 30 de setembro, p. 83-85.

Trucksess MW, Stoloff $L$ 1984. Determination of aflatoxicol and AFB1 and AFM1 in eggs. J Assoc Anal Chem 67:317320.

Trucksess MW, Stoloff L, Young K, Wyatt RD, Miller RL 1983. Aflatoxicol and aflatoxins $B 1$ and $M 1$ in eggs and tissues of laying hens consuming aflatoxin-contaminated feed. Poultry Science 62: 2176-2182.

Wyatt RD, Marks HL, Manning RO 1987. Selection for resistance to aflatoxin in chickens. Poultry Science 66:19011904. 